UVX 2008 (2009) 169-172

(C) EDP Sciences, 2009

DOI: $10.1051 / \mathrm{uvx} / 2009027$

\title{
Investigation of a laser-induced ablation plume of mercury as a nonlinear medium for Vacuum UltraViolet generation
}

\author{
L. Philippet, S. Chenais, S. Forget and M.-C. Castex \\ Laboratoire de Physique des Lasers, Institut Galilée, CNRS/Université Paris 13, 99 Av. \\ J.-B. Clément, 93430 Villetaneuse, France
}

\begin{abstract}
The efficiency of nonlinear processes in metallic vapours is limited by usually low atomic densities. One way to circumvent this limitation, while keeping simplicity (that is avoiding the use of complex and user-unfriendly heat pipes), is to use a laser-induced ablation plume of the metal as a nonlinear medium. Here we present a study of a $\mathrm{Hg}$ plume used to amplify 125 -nm radiation created by a resonant 4-wave-mixing process.
\end{abstract}

\section{INTRODUCTION}

Highly coherent, compact and reliable sources in the Vacuum UltraViolet (VUV) region $(105 \mathrm{~nm}<$ $\lambda<180 \mathrm{~nm}$ ) would be beneficial for many applications. For example, interference lithography of sub$100 \mathrm{~nm}$ patterns would require short wavelength lasers with a long coherence length, which is not the case of standard excimer lasers $\left(\mathrm{ArF}, \mathrm{F}_{2}\right)$. An alternative solution consists in using visible coherent lasers followed by nonlinear conversion in gases down to the VUV. These sources have a better coherence and beam quality but generally have very low energies (typically $<\mu \mathrm{J}$ per pulse). Since the efficiency of a 4-wave-mixing process scales as the square of the gas atomic density (notwithstanding saturation or phase-matching issues), we recently reported a significant improvement (up to 2 orders of magnitude without saturation) of the yield of a $125-\mathrm{nm}$ source based on mercury vapour by creating a localized increase of the $\mathrm{Hg}$ atoms density thanks to an ablation plume created by an external vaporizing laser [1]. We present here a complete analysis of the plume properties in order to identify the relevant parameters that will allow designing a system with optimized efficiency.

\section{EXPERIMENTAL SET UP}

The non-amplified VUV source at $125 \mathrm{~nm}$ has been described in detail in a previous publication [1]. The sum frequency signal $\left(\omega_{\mathrm{VUV}}=2 \omega_{\mathrm{UV}}+\omega_{\mathrm{VIS}}\right)$ was generated at room temperature in the atomic vapour above a mercury pool where two collinear beams at $626 \mathrm{~nm}\left(\omega_{\mathrm{VIS}}, 10 \mathrm{~Hz}-6 \mathrm{~ns}\right)$ and $313 \mathrm{~nm}\left(\omega_{\mathrm{UV}}\right)$ were focussed (fig. 1). The vaporizing laser was an ArF excimer laser emitting at $193 \mathrm{~nm}$ with an incident energy of $\approx 1 \mathrm{~mJ}$ per pulse $(10 \mathrm{~Hz}-4 \mathrm{~ns})$.

The plume was studied with two time-gated ICCD cameras (Andor iStar DH734 for imaging and DH720 for spectroscopy). Three complementary experiments were carried out (fig. 1). The first technique consisted in recording fast photographies of plume luminescence (spectral lines emitted from the excited states thermally populated during the pulse laser heating). The plume expansion was compared for different ArF laser energy density in vacuum and for different transparent background gas pressures (argon). The second technique consisted in taking advantage of the 2-photon-resonant excitation at $\omega_{\mathrm{UV}}$ to monitor the LIF (Laser Induced Fluorescence) which yields a signal scaling as the square of the ground state atomic density at low UV intensities (the only ones playing a role in the nonlinear interaction). We can deduce information about $\mathrm{Hg}$ atoms in the ground state within the interaction volume. 
At last, we added a monochromator (grating 150 lines/mm, 27-cm focal length) coupled with the camera to perform LIBS (Laser Induced Breakdown Spectroscopy) experiments. Hg species were identified and the decay of spectral lines and their relative amplitude provided useful information about the role of excited states in the phase matching of the nonlinear process.

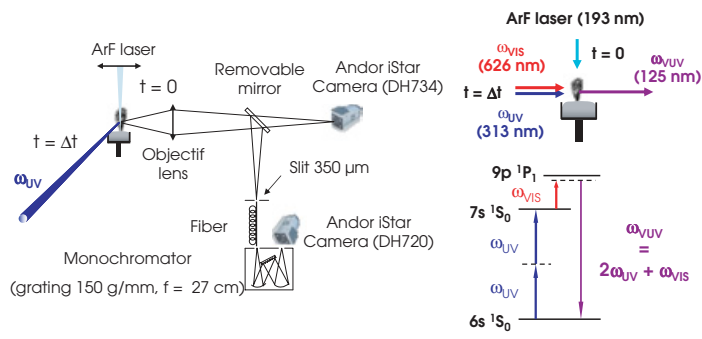

Figure 1. (left) Experimental set up for fast photographies of plume luminescence (without UV beam), time resolved spectral analysis of plume emission and 2-photon laser induced fluorescence. (right) Amplification set up and energy diagram of the 2-photon resonant nonlinear interaction.

\section{RESULTS AND DISCUSSION}

We monitored fast photographies of the plume luminescence for two different spot sizes with a constant ArF energy in vacuum (fig. 2 top) and in argon (fig. 2 bottom). In vacuum, we observed a wide angle of plume expansion when ArF laser is tightly focused $\left(0.1 \mathrm{~mm}^{2}\right.$ spot size $)$ and a "columnar" shape when spot size is bigger $\left(1 \mathrm{~mm}^{2}\right)$, resulting in a higher $\mathrm{Hg}$ density and a better VUV yield in these conditions (two times higher with $1 \mathrm{~mm}^{2}$ spot size compared to $0.1 \mathrm{~mm}^{2}$ spot size) [1]. We also added a transparent background gas (argon) in the $\mathrm{Hg}$ cell and observed a strong plume confinement effect (the VUV yield is 1.5 times higher with argon when compared to vacuum for the same focusing conditions i.e. $1 \mathrm{~mm}^{2} \mathrm{spot}$ size). Systematic study of argon pressure is in progress for optimizing the VUV amplification. Persistent fluorescence (until $10 \mu \mathrm{s}$ vs $1 \mu \mathrm{s}$ in vacuum) was observed due to enhanced collisions (fig. 2).

In vacuum, we mapped the plume luminescence intensity and deduced, from the position of the plume center versus time, a constant plume velocity of $2700 \mathrm{~m} \cdot \mathrm{s}^{-1}$. In similar experimental conditions, Bennett et al. reported plume velocity with comparable order of magnitude [3].

The 2-photon excitation $6 s{ }^{1} S_{0} \rightarrow 7 s^{1} S_{0}(\lambda=313 \mathrm{~nm})$ followed by atomic collisions between excited states creates population in the $7 s^{3} S_{1}$ and $6 s^{3} D_{2,3}$ states (fig. 3) [2]. From UV LIF images (such as the one depicted in fig. 3), for different positions of the UV beam into the plume and different delays between the ArF laser and the UV pulse, it is possible to have qualitative information about the density of atoms in the ground state (the only ones playing a role in the nonlinear interaction) as a function of space and time, thus giving a complete picture of the nonlinear medium. Quantitative analysis (i.e. estimate of density) requires further information and is already under investigation.

From time-resolved spectroscopy (fig. 4), we observed $\mathrm{Hg}$ lines superposed with a continuum at early time and only $\mathrm{Hg}$ lines after $t=20 \mathrm{~ns}$, essentially due to excited neutral $\mathrm{Hg}(\mathrm{Hg} \mathrm{I})$. The decay time of the $546.1,435.8$ and $404.7 \mathrm{~nm}$ lines originating from $7 s^{3} S_{1}$ state is higher $(46 \mathrm{~ns})$ than natural radiative lifetime ( $8 \mathrm{~ns}$ ) (see inset fig. 4). Such a slow $7 s^{3} S_{1}$ population decay can be explained by cascading from higher excited levels and collisions, which populates the $7 s^{3} S_{1}$ state at longer times [2]. Experimentally, we noticed that the best VUV enhancement was obtained when the ArF pulse hit the $\mathrm{Hg}$ surface $\approx 1 \mu$ s before the UV and visible pulses (fig. 5), thus well after the plume luminescence has ceased. This optimum time delay is not directly linked to the population of excited states. $\mathrm{Hg}$ I lines reappear when the UV beam pass through the plume because of the 2-photon excitation at $313 \mathrm{~nm}$.

In order to understand the connection between the plume density and the VUV signal, we measured the total LIF intensity (from images similar to fig. 3 with $100 \mathrm{~ns}$ integration time) and the VUV 


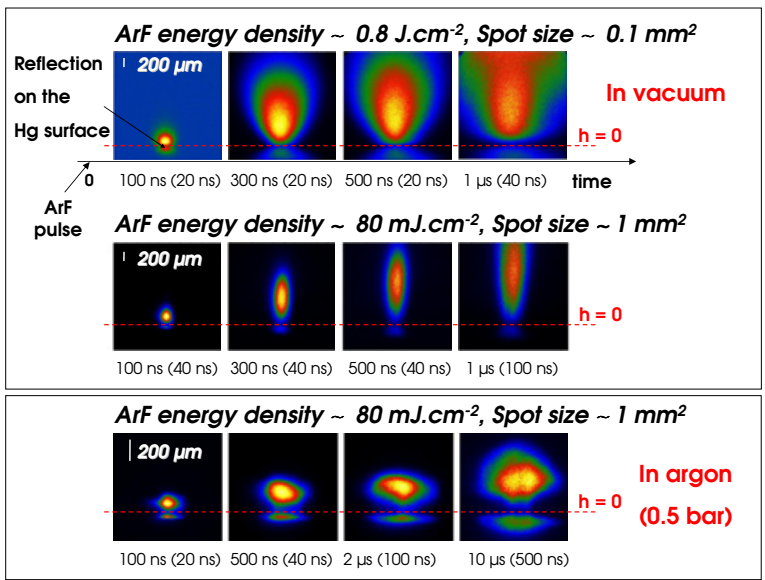

Figure 2. Fast photographies of the plume luminescence in vacuum for two different ArF energy density (top) and in 0.5 bar of argon (bottom). The ArF laser pulse hits the $\mathrm{Hg}$ surface at $\mathrm{t}=0$. Each image is normalized, into brackets the integration time.

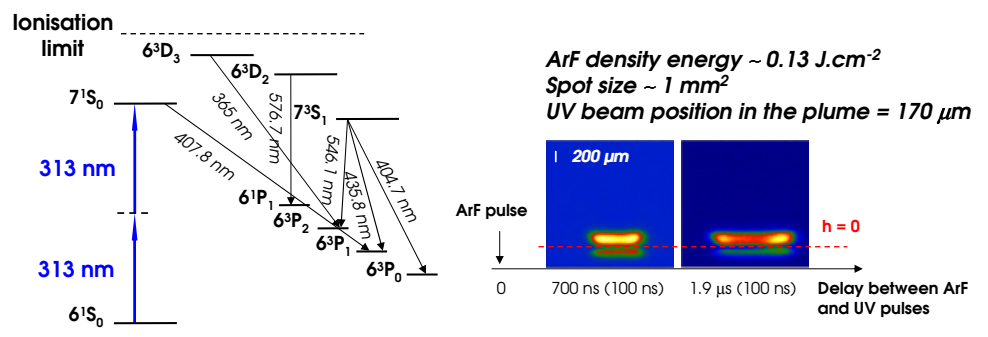

Figure 3. (left) $\mathrm{Hg}$ spectral lines emitted from the $7 s^{3} S_{1}$ and $6 s^{3} D_{2,3}$ states after the 2-photon excitation $6 s{ }^{1} S_{0} \rightarrow 7 s^{1} S_{0}(\lambda=313 \mathrm{~nm})$. (rigth) Examples of images of UV LIF in vacuum. Each image is normalized, into brackets the integration time.

enhancement (with respect to the signal obtained in room-temperature mercury without the ArF laser) for different heights $h$ of the UV beam into the plume (170,860 and $2120 \mu \mathrm{m})$ (fig. 5). We noticed that the optimal delay corresponding to the maximum VUV signal enhancement is almost independent of the height $h$ of the mixing beams. The LIF signal for $h=170 \mu \mathrm{m}$ shows that the number of $\mathrm{Hg}$ atoms in the ground state located in the path of the mixing beams is higher at shorter time delays, which is consistent with the plume velocity measurements. It is clear that the time delay for maximum VUV enhancement is not here linked to the time needed for the plume to reach the beams (less thans $100 \mathrm{~ns}$ ). The reason could be a phase matching alteration at early times due to too high atomic density. Further studies are needed to confirm this hypothesis.

\section{CONCLUSION}

Combination of space and time-resolved analysis of the optical emission of a $\mathrm{Hg}$ laser-induced ablation plume is a powerful tool to understand in which conditions a dense nonlinear medium for efficient VUV generation can be obtained. The physical origin of such a long time delay between plume creation and maximum VUV efficiency is not straightforwardly linked to the time-of-flight of the ejected material 


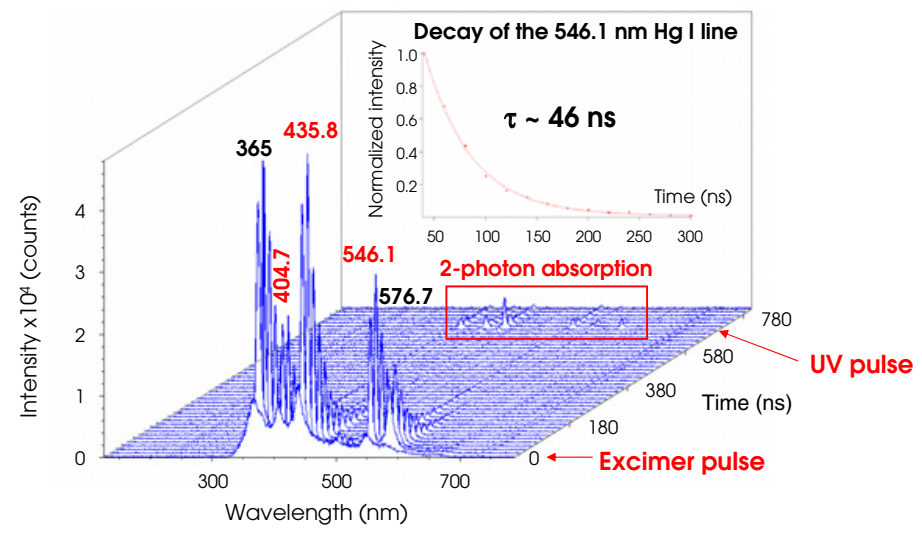

Figure 4. Plume spectra (in vacuum) from $t=0 \mathrm{~ns}$ to $t=820 \mathrm{~ns}$, integration time $=20 \mathrm{~ns}$, ArF energy density $0.1 \mathrm{~J} \cdot \mathrm{cm}^{-2}$. (inset) Decay of the $546.1 \mathrm{~nm} \mathrm{Hg} \mathrm{I}$ line.

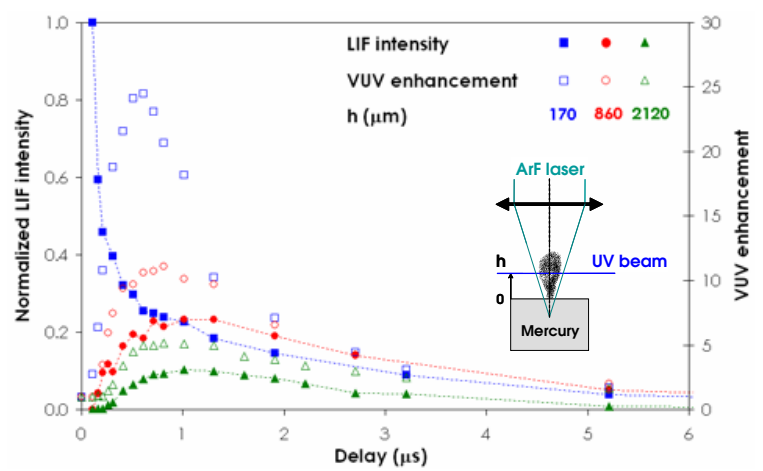

Figure 5. LIF intensity and VUV enhancement vs delay for 3 differents positions $h(170,860$ and $2120 \mu \mathrm{m})$ of the UV beam in the plume (ArF energy density $\left.\sim 0.13 \mathrm{~J} \cdot \mathrm{cm}^{-2}\right)$.

up to interaction volume (ruled out by LIF and velocity measurements) neither to the time needed for recovering a high density of ground state atoms after excited species have recombined (ruled out by time-resolved spectral analysis and LIF). Work is in progress for a systematic study of argon pressure (where higher energy was measured) and quantitative measurements of output energies.

We gratefully acknowledge Andor Technology for loaning us the imaging camera.

\section{References}

[1] S. Chénais and al., Applied Phys B 89, (2007) 223-229.

[2] N. Bras and al., J. Phys. B: At. Mol. Opt. Phys. 26, (1993) 2289-2302.

[3] T. D. Bennett and al., J. Heat Transfer 122, (2000) 345-350. 